\title{
Human Immunodeficiency Virus and Human Papillomavirus Co-Infection in Pregnant Woman
}

\author{
Anna Kornete*, Elizabete Pumpure*, Jana Zodzika*,**, Dace Rezeberga*,**,***, \\ Liga Puksta - Gulbe*,**, Irina Jermakova*,** \\ * Riga Stradins University, Latvia \\ ** Riga East Clinical University Hospital, Latvia \\ *** Riga Maternity Hospital, Latvia
}

\begin{abstract}
Summary condyloma acuminata in association with vaginal bleeding during pregnancy.

Key words: cervical cancer, condyloma acuminata, pregnancy, hysterectomy

\section{AIM OF THE DEMONSTRATION}

Latvia is the country with one of the highest HIV infection incidence rates in Europe with 16.8 cases per 100,000 population (2). Most of the women living with HIV/AIDS are in reproductive age (2). Persistent HPV infection is significantly higher in HIV-infected women compared with HIV-uninfected, $20-30 \%$ and 5-10 \%, respectively. Moreover, multiple HPV genotypes are prevalent, and viral load is higher (4). As a result, cervical cancer incidence is 6-10 times increased (4). A clinical case of HIV and HPV co-infection demonstrates woman with invasive cervical cancer and cervical and vaginal condyloma acuminata with vaginal bleeding during pregnancy.
\end{abstract}

Among HIV-infected women prevalence and incidence of HPV infection are higher, and the risk for developing cervical cancer is increased. We present a case of HIV and HPV co-infection in woman with invasive cervical cancer and cervical and vaginal

\section{CASE REPORT}

We report a case of a 29yearold primiparous woman with HIV/AIDS diagnosed within screening programme at the beginning of pregnancy. The patient did not belong to high-risk group for sexually transmitted infections. There were no identifiable risk factors for HIV infection before the pregnancy. After the diagnosis of HIV/AIDS was made, for the first time it was found out that the patient's husband has been intravenous drug user in the past. The patient's CD4 Tlymphocytes count was 317 cells $/ \mathrm{mm} 3$, HIV viral load was 20,000 copies $/ \mathrm{ml}$. Antiretroviral therapy with lamivudine/zidovudine and lopinavir/ritonavir was initiated. No abnormalities were found in cervical cytology at 8th weeks gestation.

At 29th week the patient presented with severe vaginal bleeding, which was interpreted as imminent preterm delivery. Gynecologic examination revealed cervical and vaginal condyloma acuminata with erosive cervical lesion. During transvaginal sonography, there were no convincing evidence for invasive cervical cancer. In colposcopicaly directed cervical punch biopsy highgrade squamous intraepithelial lesion with features suspicious for invasion was observed (Figure 1). Therefore, an elective Cesarean section was performed at the $34^{\text {th }}$ week of gestation.
Cervical punch biopsy performed after delivery confirmed moderately differentiated large cell nonkeratinizing squamous cell carcinoma. A CT scan of the pelvis revealed a cervical mass $4.5 \mathrm{~cm}$ in diameter (according to the FIGO stage IB2) (Figure 2). Eight weeks post-partum pelvic radiotherapy with 50.4 Gy was conducted followed by Wertheim's hysterectomy with bilateral salpingectomy. No HIV viral load in twomonths-old infant was detected.

\section{DISCUSSION}

The role of pregnancy in the carcinogenesis of cervical cancer is still incomprehensible. Physiological changes of pregnancy due to immunosuppression, hypervascularisation and significant changes in hormonal homeostasis have been postulated as contributing factors for worse outcome $(1,5)$. Moreover, physiological changes of the cervix complicate the interpretation of cytological analysis, hence raising difficulties in cervical cancer diagnostics (3). Women with multiple both lowand high-risk HPV types infection resulting in cervical condyloma acuminata and cervical erosion are exposed to increased risk for the development of cervical malignancy (6). Finally, HPV-induced lesions as cervical cancer, cervical and vaginal condyloma acuminata have a proclivity to increase in size and to be more aggressive due to HIV-associated immune suppressive state (4).

Vaginal bleeding during pregnancy in most cases is associated with pregnancy, including placenta previa or placental abruption, though it also might be caused by cervical malignancy, particularly in immunocompromised women. Although diagnosis of gynecologic oncology during pregnancy is uncommon, cervical cancer in pregnancy should be kept in mind when pregnant woman presents with vaginal bleeding (6).

In conclusion, HPV infection is particular burden for HIV-infected women, increasing the risk of developing different types of HPV-associated diseases. Moreover, in HIV-infected women squamous cell carcinoma tends to 
respond to recommended therapies poorly and has an overall worse prognosis (3).

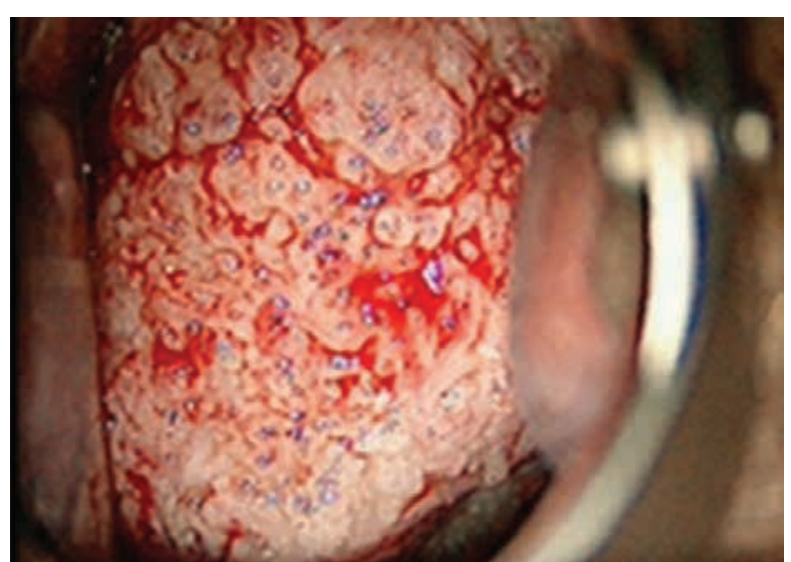

Fig. 1. Colposcopic visualization with

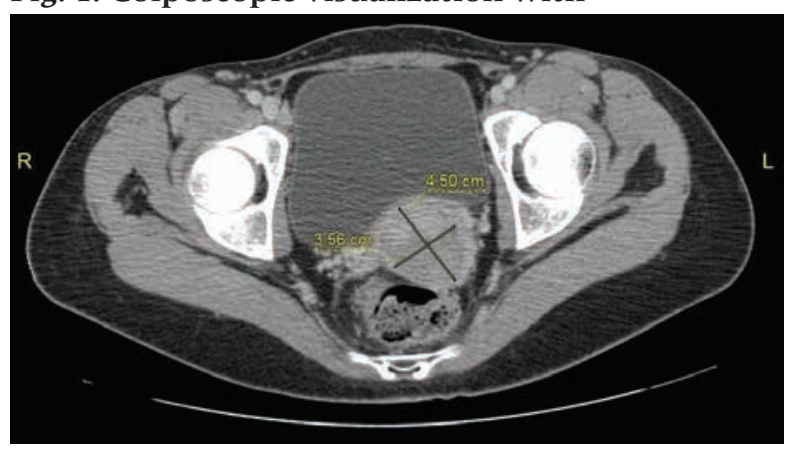

Fig. 2. A CT scan revealing a mass $4.5 \mathrm{~cm}$ in features suspicious for invasion diameter in the cervix

\section{Conflict of interest: None}

\section{REFERENCES}

1. Amant F, Halaska MJ, Fumagalli ZM, et al. Gynecologic Cancers in Pregnancy. Guidelines of a Second International Consensus Meeting // Int J Gynecol Cancer 2014; 24:394 - 403

2. European Centre for Disease Prevention and Control. HIV and hepatitis B and C in Latvia: ECDCEMCDDA joint technical mission, 2 - 4 September 2014. Stockholm: ECDC; 2015

3. Freitas BC, Suehiro TT, Consolaro M, Silva V. HPV Infection and Cervical Abnormalities in HIV Positive Women in Different Regions of Brazil, a MiddleIncome Country // Asian Pac J Cancer Prev 2015; 16 (18): $8085-8091$

4. Konopnicki D. HPV-related cancers and their prevention in women: WAVE Workshop Against Viruses in Europe - Promoting the Welfare of HIVpositive Women in Europe Barcelona, Spain; 2015

5. Morice P, Uzan C, Gouy S, et al. Gynaecological cancers in pregnancy // Lancet 2012; 379:558 - 569

6. Yao J, Cao X, Gao N, et al. Correlation of subclinical HPV infection with genital warts and cervical erosion // Eur J Gynaecol Oncol 2013; 34(5):462 - 5

\section{Address:}

Anna Kornete

Valmieras street 7 Cesis, Latvia, LV-4101

E-mail: annakornete@gmail.com 